\title{
Periodontitis is associated with rheumatoid arthri- tis: a study with longstanding rheumatoid arthritis patients in Korea
}

In Ah Choi ${ }^{1}$, Jin-Hee Kim² ${ }^{2}$ Yong Mi Kim², Joo Youn Lee³ ${ }^{3}$ Kyung Hwa Kim²,4, Eun Young Lee Eun Bong Lee ${ }^{1}$, Yong-Moo Lee ${ }^{2,4}$, and Yeong Wook Song ${ }^{1,3}$

\begin{abstract}
${ }^{1}$ Department of Internal Medicine, Medical Research Center, Seoul National University College of Medicine, Seoul; '2Department of Periodontology, Seoul National University School of Dentistry, Seoul; ${ }^{3}$ Department of Molecular Medicine and Biopharmaceutical Sciences, Seoul National University, Seoul; ${ }^{4}$ Dental Research Institute, Seoul National University School of Dentistry, Seoul, Korea
\end{abstract}

Received: July 3, 2015

Revised : August 28, 2015

Accepted: September 2, 2015

\section{Correspondence to}

Yeong Wook Song, M.D.

Department of Internal Medicine, Seoul National University College of Medicine, 101 Daehak-ro, Jongno-gu, Seoul 03080, Korea

Tel: $+82-2-2072-2234$

Fax: $+82-2-762-9662$

E-mail:ysong@snu.ac.kr
Background/Aims: A cross-sectional study was undertaken to investigate the association between severity of periodontitis and clinical manifestation of rheumatoid arthritis (RA).

Methods: Two hundred sixty-four RA patients and 88 age- and sex-matched controls underwent dental exam. Additionally, clinical manifestations including disease activity and anti-citrullinated protein antibodies were evaluated in RA patients.

Results: The prevalence of moderate or severe periodontitis was higher in RA patients compared to controls (63.6\% vs $34.1 \%, p<0.001)$. In markers of periodontal inflammation, bleeding on probing was correlated with disease activity score $28(r$ $=0.128, p=0.041)$, RA disease duration $(r=0.211, p=0.001)$, erythrocyte sedimentation rate (ESR; $r=0.141, p=0.023)$, anti-cyclic citrullinated peptide antibody $(r$ $=0.183, p=0.009)$, and anti-citrullinated $\alpha$-enolase peptide- 1 antibody $(r=0.143$, $p=0.025)$. Gingival index was correlated with RA duration $(r=0.262, p<0.001)$, $\operatorname{ESR}(r=0.162, p=0.009)$, anti-cyclic citrullinated peptide antibody $(r=0.203, p=$ $0.004)$ and anti-citrullinated $\alpha$-enolase peptide-1 antibody $(r=0.225, p<0.001)$. Periodontal structural damage represented by probing pocket depth and clinical attachment level were less in RA patients with human leukocyte antigen (HLA)DRB1 shared epitope compared than those without shared epitope $(p=0.005$ and $p=0.006$, respectively).

Conclusions: The prevalence of moderate or severe periodontitis was increased in RA patients compared to controls. Periodontal inflammation was correlated with RA disease duration, ESR, and anti-citrullinated protein antibodies. Periodontal structural damage was less in RA patients with HLA-DRB1 shared epitope.

Keywords: Periodontitis; Arthritis, rheumatoid

\section{INTRODUCTION}

Antibodies against the citrullinated proteins were reported to be important in the pathogenesis of anti-ci- trullinated protein antibody-positive rheumatoid arthritis (RA) [1]. Epidemiologic studies have identified smoking as a major risk factor for developing RA more than 20 years ago $[2,3]$, and smoking has been consid- 
ered to induce the enzyme peptidylarginine deaminase (PAD), which can catalyze the conversion of arginine into citrulline during lung inflammation [4]. This may result in the formation of a new epitope and breakage of immune tolerance, causing a pathologic state called RA. However, smoking-induced lung citrullination is not sufficient to explain all cases of anti-citrullinated protein antibody-positive RA as far as there are a substantial number of non-smokers still developing RA. Another candidate risk factor is chronic infection such as periodontitis.

Periodontitis is a chronic inflammatory disease characterized by loss of the periodontal ligament and alveolar bone, and it is a major cause of tooth loss. Pathogenic processes in RA, which is characterized by synovial inflammation and progressive destruction of cartilage and bone, are similar to those in periodontitis. Also, epidemiologic studies have demonstrated that the prevalence of periodontitis is increased in RA population compared to the healthy subjects [5-9], and patients with periodontitis have a higher prevalence of RA than patients without periodontitis [10]. To explain this relationship, the bacterium Porphyromonas gingivalis, one of the major pathogens in periodontitis received great attention. Successive studies showed that PAD produced by $P$. gingivalis can citrullinate human peptide [11], and there is a significant correlation between anti- $P$. gingivalis antibody titer and anti-cyclic citrullinated peptide (CCP) antibodies [12]. Moreover, antibodies directed towards the immunodominant epitope of human citrullinated $\alpha$-enolase were reported to cross react with a conserved sequence on citrullinated P. gingivalis enolase [13]. However, no consistent association between anti-citrullinated protein antibody status [14-16] or disease activity [16-18] of RA with periodontitis were demonstrated in previous studies. Therefore, we investigated the prevalence and severity of periodontitis and its associated factors including disease activity, human leukocyte antigen (HLA)-DRB1 shared epitope, and anti-citrullinated protein antibodies including anti-CCP antibody, anti-citrullinated $\alpha$-enolase peptide- 1 (anti-CEP-1) antibody in Korean patients with RA.

\section{METHODS}

\section{Patients}

We conducted a prospective, cross-sectional study comparing RA patients with controls. RA patients who satisfied the 1987 American College of Rheumatology classification criteria for RA and age- and sex-matched control group without arthritis were enrolled in a 3:1 ratio at the rheumatology clinic of Seoul National University Hospital from May 2011 to February 2012. The Institutional Review Board and ethics committee approved the protocol (H-1103-151-357), and written informed consent was obtained from each patient and control before enrollment in the study.

\section{Study design}

Patient's data such as socio-demographic data, medical history, and comorbidities were collected. In RA patients, clinical parameters including 68 tender joint count (TJC), 66 swollen joint count (SJC), disease duration and medication were evaluated and erythrocyte sedimentation rate (ESR) and serum C-reactive protein (CRP) were measured. Disease activity score 28 (DAS28) was calculated as $[0.56 \times \sqrt{ }(28 \mathrm{TJC})+0.28 \times \sqrt{ }(28 \mathrm{SJC})$ $+0.70 \times \ln (E S R)] \times 1.08+0.16$ [19]. Serum rheumatoid factor (RF), anti-CCP antibody, anti-CEP-1 antibody, anti-arginine-containing $\alpha$-enolase peptide 1 (anti-REP-1) antibody, presence of HLA-DRB1 shared epitopes, and radiographic erosive changes were evaluated at the time point of obtaining samples.

\section{Laboratory procedures}

Anti-CEP-1 and anti-REP-1 antibodies were measured by enzyme-linked immunosorbent assay. CEP-1 (KIHA-Cit-EIFDS-Cit-GNPTVE) [20] and REP-1 (KIHAR-EIFDS-R-GNPTVE) was synthesized by Peptron (Daejeon, Korea). The 96-well plates (MaxiSorp, Nunc, Roskilde, Denmark) were coated with CEP-1 peptide or REP-1 peptide at $2 \mu \mathrm{g} / \mathrm{mL}$ (diluted in a $50 \mathrm{mM}$ carbonate buffer, $\mathrm{pH}$ 9.6), and incubated overnight at $4^{\circ} \mathrm{C}$. After washing three times with phosphate-buffered saline (PBS) containing $0.05 \%$ Tween20 ( $\mathrm{pH} 7.4$ ) and blocking with PBS containing $1 \%$ bovine serum albumin, 2-fold serial dilutions of serum from patients and controls (first dilution 1:200) were added to the plate and the bound human immunoglobulin G (IgG) was 
detected with Horseradish peroxidase-conjugated, gout anti-human IgG antibodies (Millipore, Billerica, MA, USA; $1 / 6,000$ dilution), followed by the addition of a developer containing TMB (KPL, Gaithersburg, MD, USA). The anti-CEP-1 antibody and anti-REP-1 antibody titers were determined as the inverse value of the largest serial dilution for which detectable antibody was observed. Antibody titers above the 95th percentile of healthy controls were arbitrarily considered as positive.

Serum RF were measured by the immunoturbidimetry method (Roche, Basel, Switzerland), and anti-CCP antibody was measured by chemiluminescent microparticle immunoassay (Abbott, Lake Bluff, IL, USA) according to the manufacturer's instructions. Anti-CCP antibody titer over 5 arbitrary units $/ \mathrm{mL}$ was considered as positive.

For high resolution HLA-DRB1 subtyping, group-specific amplification and single-strand conformation polymorphism analysis were performed, as described by Bannai et al. [21], using specific primers and by hybridization with sequence-specific oligonucleotides for HLA-DRB1 0101, 0102, 0103, 1001, 0401, 0403, 0404, 0405, 0406, 0407, 0408, and 0410. Shared epitopes were determined, if the HLA-DR type was one of following; HLA-DRB1 0101, 0102, 1001, 0401, 0404, 0405, 0408, and 0410 [22].

\section{Dental examination}

In all of the subjects, the number of teeth (o to 28, excluding 3rd molars) was checked. Among the 295 RA patients, the periodontal status was evaluated in 264 patients by dentists specialized in periodontology. Individual with small number of teeth (less than 15) or ongoing dental care were excluded because they could cause bias in evaluation of overall severity of periodontitis. Plaque index (PI) was evaluated as a marker of dental hygiene and was graded as 1, 2, and 3 (higher PI represented poor dental hygiene). Measurements were taken at 4 sites (three buccal points and one lingual point) of each tooth and the mean values (from maximum 112 sites in 32 teeth) were used for calculating the PI [23].

Bleeding on probing (BOP), gingival index (GI), probing pocket depth (PPD), and clinical attachment level (CAL) were used as the indices of periodontitis. Among them, BOP and GI reflected periodontal inflammation whereas PPD and CAL reflected periodontal structural damage.
BOP was assessed as positive/negative (coded into 1/0) and positive BOP represented an early sign of inflammation. Mean value measured at six sites in each tooth was presented as the percentage and used as the BOP in the patient. GI was graded as 1, 2, and 3, and a higher index represented more inflammation of the gingiva [24]. Measurements were taken at four sites (three buccal points and one lingual point) of each tooth, and mean values of maximum 112 sites were used for calculating the GI. PPD was measured by using the $15 \mathrm{~mm}$-University of North Carolina (UNC) probe in $\mathrm{mm}$ scale, and a higher index represented more severe structural changes. PPD was measured at six sites (three buccal points and three lingual points) in each tooth, and the mean values of maximum 168 sites were used for calculating the PPD. PPD values more than $4 \mathrm{~mm}$ were considered to indicate the presence of a pathologic condition.

CAL is the distance from the cementoenamel junction to the base of the probable crevice. It was calculated as sum of PPD and gingival recession measured with $15 \mathrm{~mm}$-UNC probe in $\mathrm{mm}$ scale. Mean value calculated at 6 sites in each tooth was used as the CAL for the patient, and CAL was regarded as the practical index of periodontitis. The severity of periodontitis was defined as slight (CAL 1 to $2 \mathrm{~mm}$ ), moderate (CAL 3 to $4 \mathrm{~mm}$ ), and severe (CAL $\geq 5 \mathrm{~mm}$ ) according to American Academy of Periodontology classification [25].

\section{Statistical analyses}

Statistical analysis of nonparametric variables was performed with IBM SPSS version 19.0 (IBM Co., Armonk, NY, USA). The correlations between periodontal indices and RA disease activity/characteristics were analyzed by Pearson or Spearman correlation coefficients, as appropriate. Baseline data were presented as mean $\pm \mathrm{SD}$ or percentages (\%) and analyzed by Student $t$ test or chisquare test. Mann-Whitney U test was applied for analysis of independent nonparametric variables. All $p$ values are two-sided, and $p$ values less than 0.05 were considered statistically significant.

\section{RESULTS}

\section{Baseline characteristics of RA patients and controls}

The mean number of teeth in 295 RA patients and 88 
Table 1. Baseline characteristics of rheumatoid arthritis patients and controls

\begin{tabular}{|c|c|c|c|}
\hline Characteristic & $\mathrm{RA}(\mathrm{n}=264)$ & Control $(\mathrm{n}=88)$ & $p$ value \\
\hline Age, yr & $58.2 \pm 12.0$ & $58.2 \pm 11.6$ & 0.992 \\
\hline Female sex, \% & 87.5 & 87.5 & 1.000 \\
\hline Ever smokers, \% & 6.4 & 8.0 & 0.625 \\
\hline Alcohol users, \% & 17.0 & 25.0 & 0.268 \\
\hline Steroid users, \% & $47 \cdot 3$ & o & $<0.001$ \\
\hline NSAID users, \% & 80.7 & o & $<0.001$ \\
\hline Biologic agent users, \% & 11.4 & o & $<0.001$ \\
\hline \multicolumn{4}{|l|}{ Comorbidity, \% } \\
\hline Hypertension & 16.9 & 17.0 & 0.810 \\
\hline Diabetes mellitus & $5 \cdot 6$ & 2.3 & 0.262 \\
\hline Disease duration, yr & $13.8 \pm 9.8$ & & \\
\hline Presence of sicca syndrome, \% (n) & $6.8(18 / 264)$ & & \\
\hline DAS28 & $3.3 \pm 1.4$ & & \\
\hline RF positive, \% (n) & $68.5(176 / 257)$ & & \\
\hline Anti-CCP antibody positive, \% (n) & $69.1(158 / 226)$ & & \\
\hline Presence of HLA-DRB1 shared epitope, \% (n) & $63.4(109 / 172)$ & & \\
\hline Presence of bone erosion, \% & 65.6 & & \\
\hline
\end{tabular}

Values are presented as mean \pm SD.

RA, rheumatoid arthritis; NSAID, nonsteroidal anti-inflammatory drug; DAS28, disease activity score 28; RF, rheumatoid factor; CCP, cyclic citrullinated peptide; HLA, human leukocyte antigen.

non-arthritic controls were $23.4 \pm 6.2$ vs. $25.9 \pm 2.9$, respectively $(p<0.001)$. Twenty-eight RA patients $(8.8 \%)$ who had less than 15 teeth and three patients who were receiving ongoing dental care were excluded from further dental exam. As a result, $264 \mathrm{RA}$ and 88 controls underwent the study procedures. There were no significant differences in the patient's baseline characteristics between the two groups. The proportion of smokers was $6.4 \%$ in the RA group and $8.0 \%$ in the control group.

Mean disease duration in RA patients was $13.8 \pm 9.8$ years and mean DAS28 was $3.3 \pm 1.40$. RA patients had sicca syndrome (6.8\%), RF (68.5\%), anti-CCP antibody (69.1\%), anti-CEP-1 antibody (17.5\%), and anti-REP-1 antibody (19.1\%). Radiographic erosion was found in $65.6 \%$ of RA patients (Table 1).

\section{Comparison of periodontal indices between RA patients and controls}

RA patients had significantly higher PI $(0.84 \pm 0.49$ vs. 0.71 $\pm 0.34, p=0.007)$, BOP $(20.1 \% \pm 15.4 \%$ vs. $12.3 \% \pm 10.9 \%$, $p<0.001)$, GI (o.51 \pm 0.43 vs. $0.16 \pm 0.19, p<0.001)$, PPD $(1.96 \pm 0.36 \mathrm{~mm}$ vs. $1.74 \pm 0.24 \mathrm{~mm}, p<0.001)$, and CAL
(3.25 $\pm 0.73 \mathrm{~mm}$ vs. $2.90 \pm 0.51 \mathrm{~mm}, p<0.001)$ compared to controls (Table 2). When RA patients were analyzed according to the severity of periodontitis, prevalence of moderate and severe periodontitis was significantly higher in RA patients compared to controls (63.6\% vs. $34.1 \%, p<0.001$; odds ratio, 3.38 ; $95 \%$ confidence interval, 2.50 to 4.57$)$.

\section{Association between dental hygiene and clinical manifestation of RA}

PI, a marker of dental hygiene, was not correlated with DAS28, TJC, SJC, RA disease duration, ESR, CRP, RF, anti-CCP antibody, anti-CEP-1 antibody, and anti-REP-1 antibody. It was also not associated with the presence of sicca syndrome, bone erosion or HLA-DRB1 shared epitope and the usage of steroids or biologic agents.

\section{Association between periodontal indices and RA disease duration}

In markers of gingival inflammation, both BOP and GI were correlated with RA disease duration $(r=0.211, p=$ 0.001 and $r=0.262, p<0.001$, respectively). In markers of 
Table 2. Dental examination results in rheumatoid arthritis patients and controls

\begin{tabular}{|c|c|c|c|}
\hline Variable & $\mathrm{RA}(\mathrm{n}=264)$ & Control $(\mathrm{n}=88)$ & $p$ value \\
\hline No. of teeth & $25.2 \pm 3.2$ & $25.9 \pm 2.9$ & 0.080 \\
\hline Plaque index & $0.84 \pm 0.49$ & $0.71 \pm 0.34$ & 0.007 \\
\hline Bleeding on probing, \% & $20.1 \pm 15.4$ & $12.3 \pm 10.9$ & $<0.001$ \\
\hline Gingival index & $0.51 \pm 0.43$ & $0.16 \pm 0.19$ & $<0.001$ \\
\hline Probing pocket depth, mm & $1.96 \pm 0.36$ & $1.74 \pm 0.24$ & $<0.001$ \\
\hline Clinical attachment loss, $\mathrm{mm}$ & $3.25 \pm 0.73$ & $2.90 \pm 0.51$ & $<0.001$ \\
\hline Severity of periodontitis & & & $<0.001^{\mathrm{a}}$ \\
\hline Slight & $96(36.4)$ & $58(65 \cdot 9)$ & \\
\hline Moderate & $161(61.0)$ & $30(34.1)$ & \\
\hline Severe & $7(2.6)$ & 0 & \\
\hline
\end{tabular}

Values are presented as mean \pm SD or number (\%). Slight periodontitis, clinical attachment loss 1 to 2 mm; moderate periodontitis, clinical attachment loss 3 to $4 \mathrm{~mm}$; severe periodontitis, clinical attachment loss $\geq 5 \mathrm{~mm}$ by American Academy of Periodontology classification [25].

RA, rheumatoid arthritis.

${ }^{a}$ p value by chi-square test (slight vs. moderate and severe).
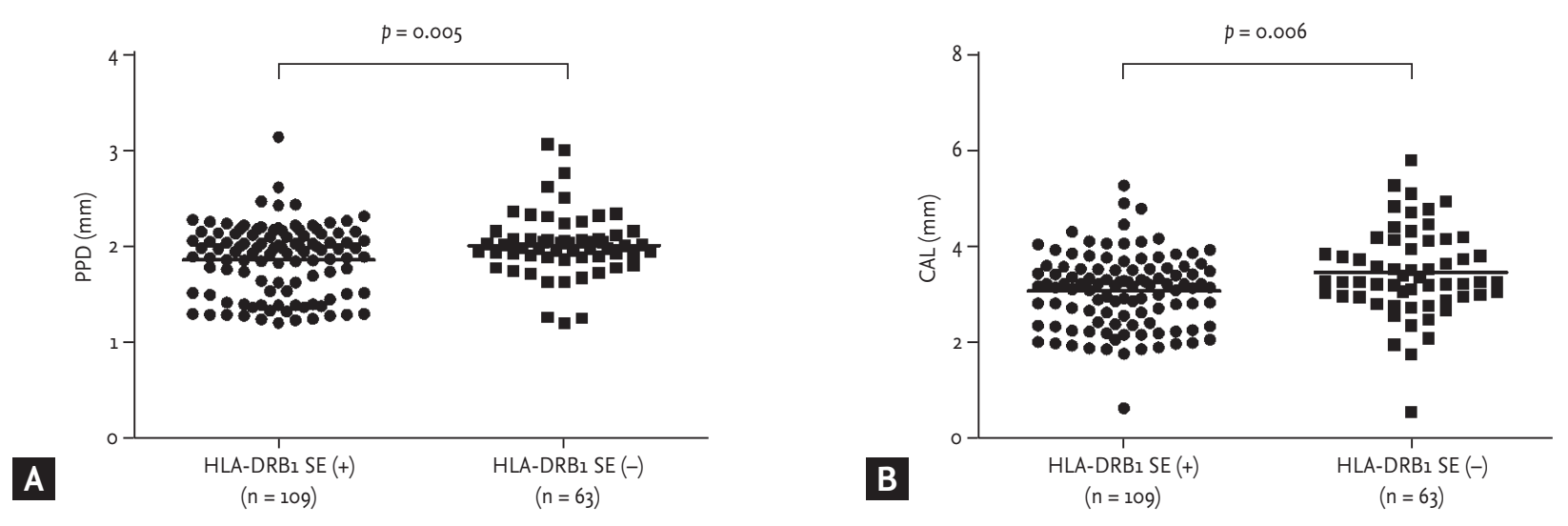

Figure 1. (A) Probing pocket depth (PPD) was significantly lower in rheumatoid arthritis (RA) patients with HLA-DRB1 shared epitope than in those without HLA-DRB1 shared epitope (mean $\pm \mathrm{SD}, 1.88 \pm 0.37 \mathrm{~mm}$ vs. $2.05 \pm 0.34 \mathrm{~mm}, p=0.005$ ). (B) Clinical attachment level (CAL) was significantly lower in RA patients with HLA-DRB1 shared epitope than in those without HLADRB1 shared epitope $(3.09 \pm 0.77 \mathrm{~mm}$ vs. $3.48 \pm 0.91 \mathrm{~mm}, p=0.006)$. Bars represent mean. SE, shared epitope.

periodontal structural damage, PPD and CAL were not correlated with RA disease duration.

Additionally, subgroup analysis based on disease duration (early RA vs. established RA vs. longstanding RA) was done. Patients with early RA (disease duration $<1$ year, $\mathrm{n}=13$ ) did not show significant difference in periodontal indices compared to the rest of the group. Patients with longstanding RA (disease duration $\geq 10$ year, $\mathrm{n}=166)$ showed significant higher GI (0.59 \pm 0.45 vs. 0.37 $\pm 0.32, p<0.001)$ and BOP $(22.7 \pm 15.7$ vs. $16.0 \pm 11.6, p<$ ०.001) compared to the rest of the group.

\section{Association between periodontal indices and clinical manifestation of RA}

In markers of gingival inflammation, BOP was correlated with DAS28 $(r=0.128, p=0.041), \operatorname{ESR}(r=0.141, p=$ $0.023)$, anti-CCP antibody $(r=0.183, p=0.009)$, and anti-CEP-1 antibody $(r=0.143, p=0.025)$ but not with TJCs, SJCs, CRP, RF, and anti-REP-1 antibody (Tables 3 and 4). BOP was not associated with the presence of sicca syndrome, bone erosion or HLA-DRB1 shared epitope or the usage of steroids or biologic agents.

GI was correlated with $\operatorname{ESR}(r=0.162, p=0.009)$, an- 
Table 3. Correlation between dental hygiene, periodontal inflammation, and periodontal structural damage with rheumatoid arthritis clinical manifestations

\begin{tabular}{|c|c|c|c|c|c|c|}
\hline Variable & DAS28 & TJC & SJC & Disease duration & ESR & CRP \\
\hline \multicolumn{7}{|c|}{ Plaque index } \\
\hline$r$ & 0.036 & 0.048 & 0.056 & 0.044 & 0.099 & 0.053 \\
\hline$p$ value & 0.607 & 0.437 & 0.368 & 0.487 & 0.108 & 0.406 \\
\hline \multicolumn{7}{|c|}{ Bleeding on probing, \% } \\
\hline$r$ & $0.128^{\mathrm{a}}$ & 0.047 & 0.004 & $0.211^{\mathrm{a}}$ & $0.141^{\mathrm{a}}$ & 0.049 \\
\hline$p$ value & 0.041 & 0.451 & 0.943 & 0.001 & 0.023 & 0.432 \\
\hline \multicolumn{7}{|c|}{ Gingival index } \\
\hline$r$ & 0.113 & 0.013 & -0.064 & $0.262^{a}$ & $0.162^{a}$ & 0.112 \\
\hline$p$ value & 0.072 & 0.832 & 0.305 & $<0.001$ & 0.009 & 0.071 \\
\hline \multicolumn{7}{|c|}{ Probing pocket depth, $\mathrm{mm}$} \\
\hline$r$ & 0.061 & 0.035 & 0.040 & 0.055 & 0.094 & 0.019 \\
\hline$p$ value & 0.334 & 0.578 & 0.518 & 0.379 & 0.809 & 0.759 \\
\hline \multicolumn{7}{|c|}{ Clinical attachment level, mm } \\
\hline$r$ & 0.054 & 0.031 & 0.019 & 0.067 & 0.072 & 0.109 \\
\hline$p$ value & 0.393 & 0.618 & 0.756 & 0.284 & 0.247 & 0.077 \\
\hline
\end{tabular}

DAS28, disease activity score 28; TJC, tender joint count; SJC, swollen joint count; ESR, erythrocyte sedimentation rate; CRP, C-reactive protein.

${ }^{\mathrm{a} C o r r e l a t i o n}$ is significant at the $p<0.05$ level.

Table 4. Correlation between dental hygiene, periodontal inflammation, and periodontal structural damage with titers of autoantibodies in rheumatoid arthritis

\begin{tabular}{|c|c|c|c|c|}
\hline Variable & Rheumatoid factor & Anti-CCP antibody & Anti-CEP-1 antibody & Anti-REP-1 antibody \\
\hline \multicolumn{5}{|c|}{ Plaque index } \\
\hline$r$ & -0.056 & 0.036 & 0.061 & -0.035 \\
\hline$p$ value & 0.375 & 0.607 & 0.341 & 0.595 \\
\hline \multicolumn{5}{|c|}{ Bleeding on probing, $\%$} \\
\hline$r$ & 0.018 & $0.183^{\mathrm{a}}$ & $0.143^{\mathrm{a}}$ & 0.025 \\
\hline$p$ value & 0.772 & 0.009 & 0.025 & 0.703 \\
\hline \multicolumn{5}{|c|}{ Gingival index } \\
\hline$r$ & 0.040 & $0.203^{\mathrm{a}}$ & $0.225^{\mathrm{a}}$ & -0.016 \\
\hline$p$ value & 0.531 & 0.004 & $<0.001$ & 0.803 \\
\hline \multicolumn{5}{|c|}{ Probing pocket depth, mm } \\
\hline$r$ & -0.060 & 0.065 & 0.015 & -0.024 \\
\hline$p$ value & 0.342 & 0.357 & 0.809 & 0.715 \\
\hline \multicolumn{5}{|c|}{ Clinical attachment level, mm } \\
\hline$r$ & -0.062 & 0.007 & 0.058 & -0.031 \\
\hline$p$ value & 0.327 & 0.917 & 0.357 & 0.632 \\
\hline
\end{tabular}

CCP, cyclic citrullinated peptide; CEP-1, citrullinated $\alpha$-enolase peptide-1; REP-1, arginine-containing $\alpha$-enolase peptide-1.

${ }^{a}$ Correlation is significant at the $p<0.05$ level. 
ti-CCP antibody $(r=0.203, p=0.004)$ and anti-CEP-1 antibody $(r=0.225, p<0.001)$. GI tended to be correlated with DAS28 $(r=0.113, p=0.072)$, but was not associated with the presence of sicca syndrome, bone erosion or HLA-DRB1 shared epitope or the usage of steroids or biologic agents.

In markers of periodontal structural damage, PPD was not correlated with DAS28, TJC, SJC, ESR, CRP, RF, anti-CCP antibody, anti-CEP-1 antibody, and anti-REP-1 antibody. PPD was not associated with the presence of sicca syndrome, bone erosion or the usage of steroids or biologic agents. However, PPD was significantly lower in RA patients with HLA-DRB1 shared epitope than in those without HLA-DRB1 shared epitope $(1.88 \pm 0.37$ $\mathrm{mm}[\mathrm{n}=109]$ vs. $2.05 \pm 0.34 \mathrm{~mm}[\mathrm{n}=63]$, respectively; $p=$ 0.005) (Fig. 1A).

CAL was not correlated with DAS28, TJC, SJC, ESR, RF, anti-CCP antibody, anti-CEP-1 antibody, and anti-REP-1 antibody. CAL was not associated with the presence of sicca syndrome, bone erosion or the usage of steroids or biologic age nts. However, CAL was significantly lower in RA patients with HLA-DRB1 shared epitope than in those without HLA-DRB1 shared epitope $(3.09 \pm 0.77$ $\mathrm{mm}[\mathrm{n}=109]$ vs. $3.48 \pm 0.91 \mathrm{~mm}[\mathrm{n}=63]$, respectively; $p=$ 0.006) (Fig. 1B).

\section{DISCUSSION}

In this study, we found that prevalence and severity of periodontitis were increased in RA patients compared to non-arthritis controls in Korean population. The association between RA and periodontitis implies that certain features of the inflammatory response might be common to both diseases, and these associations can be broadly categorized into the two possibilities that periodontitis is a causal factor in the pathogenesis of RA or that RA is a causal factor in the pathogenesis of periodontitis.

The presence of $P$. gingivalis, which is a common pathogen of periodontitis and can secrete the enzyme $\mathrm{PAD}$ is the major candidate to explain the possibilities that periodontitis is a causal factor in the pathogenesis of RA. There is experimental evidence that $P$. gingivalis oral infection can aggravate arthritis in a collagen antibody-induced arthritis [26] and a collagen-induced ar- thritis model [27]. Bacterial PAD secreted by P. gingivalis was reported to cross-react with human peptide and to citrullinate the human fibrinogen and $\alpha$-enolase [11]. As inflammatory conditions in the lungs of cigarette smokers were suggested to contribute to the breakdown of immune tolerance to citrullinated epitopes, chronic exposure to citrullinated proteins in patients with periodontitis may also predispose susceptible individuals to the development of autoantibodies and the initiation of RA. In this study, we demonstrated that antibodies against the citrullinated protein are more abundant in patients with more severe periodontal inflammation. Our results suggest that periodontitis may provide a novel source of antigenic peptides, citrullinated by bacterial PAD, inducing molecular mimicry. Although the anti-CEP-1 antibody titer showed a significant correlation with gingival inflammation, only $17.5 \%$ of RA patients had anti-CEP-1 antibody. Therefore, there may be a diverse type of citrullinated epitopes. In a recent study investigating titers of anti-CCP antibody, anti-CEP-1 antibody, anti-citrullinated vimentin, and anti-citrullinated fibrinogen in non-RA individuals, those with periodontitis showed a significant higher antibody response than those without periodontitis [28]. This finding suggests that citrullinated proteins break tolerance in periodontitis with epitope spreading in a proportion of patients that may evolve into RA.

On the other hands, the presence of RA might also predispose individuals to periodontitis. Several mechanisms were expected to result in increased periodontitis in the patients with RA including the presence of sicca syndrome, the use of medications such as corticosteroids or immunosuppressant and loss of manual dexterity required for oral hygiene practices. However, there was no statistically significant difference of periodontal indices in patients with sicca syndrome, corticosteroid treatment and biologic treatment compared to those without in our study. Also, markers of disease activity did not show the correlation with PI, the marker of dental hygiene.

Because the severity of gingival inflammation represented by BOP and GI was correlated with higher ESR and longer disease duration in this study, we could suggest that systemic inflammation in RA may affect the degree of periodontal inflammation. Therefore, subjects with severe periodontitis, or subjects with severe in- 
volvement in some teeth were recommended to receive dental treatment.

Conventional treatment recommendation of periodontitis is based on scaling and periodontal root planing. After 1 to 3 months, PPDs are checked to determine treatment response. Most of the patients need only preventive cares including brushing, smoking cessation, and regular dental visits. In some patients with severe involvement or poor treatment response, additional procedures such as resection and grafting can be done. Intervals of patient surveillance are determined by patient's risk factors and patients with the systemic disease prone to have periodontal structural damage such as RA are recommended to visit at every 1 to 3 months [29]. RA disease activity showed correlation with BOP in terms of DAS28. However, BOP was not correlated with TJC, SJC, and CRP, which are objective RA activity measures as well. In addition, the strength of correlation shown as $p$ value was not strong enough. Furthermore, GI did not show significant correlation with DAS28 and other objective RA activity measures such as TJC, SJC, and CRP. Therefore, our data was not strong enough to say that the severity of gingival inflammation correlate with RA disease activity.

HLA-DRB1 shared epitope is a risk factor for RA and is known to be associated with joint erosion in RA [30]. Interestingly, RA patients carrying HLA-DRB1 shared epitope showed less severe periodontal structural changes as represented by lower PPD and CAL while they showed no difference in periodontal inflammation. To the best of our knowledge, there has been no report about the protective effects of HLA-DRB1 in the periodontal disease assessed by detailed dental examination suggesting that patients carrying HLA-DRB1 shared epitope would have different pathophysiologic process in developing periodontitis. There was no significant association between the radiographic joint erosion and periodontal structural changes in the current study.

There are limitations to this study. First, we checked the number of teeth in all of the subjects, and we found out there was a significant difference in the number of teeth between RA patients and controls. Patients with less than 15 teeth or who were receiving ongoing dental treatment were excluded from the evaluation for calculating the accurate periodontal indices. Therefore, most advanced periodontitis patients were excluded from this analysis, and this could result in underestimation of periodontal destruction among participants.

Second, HLA-DRB1 shared epitope status was not evaluated in control group. Although we found an interesting result that RA patients with HLA-DRB1 showed less periodontal structural damage, it is hard to explain the mechanism of this finding. HLA-DRB1 is a risk factor of aggressive RA. Therefore, evaluations in non-arthritis controls would have been valuable to understand the role of HLA-DRB1 in periodontal structural damage without confounding effects of RA.

This study is the first report about the association of periodontal disease with RA in Korean population. In this cross sectional study, 264 RA patients and 88 ageand sex-matched controls were assessed by the rheumatologists and dentists specialized in periodontology. Mikuls et al. [16] reported that presence of periodontitis was more common in patients with anti-citrullinated protein antibody positive RA than in controls and it was associated with greater disease activity according to the DAS28 using CRP. Our results were similar to these findings. However they evaluated the severity of periodontitis using CAL only among variable periodontal indices. They divided patients to periodontitis or non-periodontitis group. Patients in non-periodontitis group in his study $(\mathrm{CAL}<5)$ may not be free of periodontal structural change and could have slight or moderate periodontitis [25]. We assessed the activity and severity of periodontitis in continuous variables and analyzed their correlation with clinical features of RA.

Interestingly, the proportion of ever-smokers was low in both RA patients and healthy controls $(6.4 \%$ vs. $8.0 \%$ ) of our cohort compared to the previous report in European countries. In an international cross-sectional study about comorbidities in RA, the prevalence of current smoker (recent 3 years) in Korea was $9 \%$, which was much lower than European countries (19\% to 48\%) [31]. This characteristics of our cohort would be appropriate to explain substantial number of non-smokers still developing RA, suggesting periodontitis as a novel source of anti-citrullinated protein antibodies.

In conclusion, the prevalence of moderate or severe periodontitis was increased in RA patients compared to controls. We found correlations of ESR or anti-citrullinated protein antibodies with periodontal inflammation and of RA disease duration and HLA-DRB1 shared 
epitope with periodontal structural damages in RA. Periodontitis may provide a novel source of citrullinated proteins that can induce an anti-citrullinated protein antibodies response in RA.

\section{KEY MESSAGE}

1. The prevalence of moderate or severe periodontitis was increased in rheumatoid arthritis (RA) patients compared to controls.

2. Periodontal inflammation was correlated with RA disease duration, erythrocyte sedimentation rate and anti-citrullinated protein antibodies.

\section{Conflict of interest}

No potential conflict of interest relevant to this article was reported.

\section{Acknowledgments}

This research was supported by a grant from the Ministry of Science, ICT and Future Planning (NRF2015M3A9B6052011).

\section{REFERENCES}

1. Cantaert T, De Rycke L, Bongartz T, et al. Citrullinated proteins in rheumatoid arthritis: crucial...but not sufficient! Arthritis Rheum 2006;54:3381-3389.

2. Heliovaara M, Aho K, Aromaa A, Knekt P, Reunanen A. Smoking and risk of rheumatoid arthritis. J Rheumatol 1993;20:1830-1835.

3. Silman AJ, Newman J, MacGregor AJ. Cigarette smoking increases the risk of rheumatoid arthritis: results from a nationwide study of disease-discordant twins. Arthritis Rheum 1996;39:732-735.

4. Perry E, Kelly C, Eggleton P, De Soyza A, Hutchinson D. The lung in ACPA-positive rheumatoid arthritis: an initiating site of injury? Rheumatology (Oxford) 2014;53:19401950.

5. Pischon N, Pischon T, Kroger J, et al. Association among rheumatoid arthritis, oral hygiene, and periodontitis. J Periodontol 2008;79:979-986.

6. Mercado FB, Marshall RI, Klestov AC, Bartold PM. Relationship between rheumatoid arthritis and periodontitis.
J Periodontol 2001;72:779-787.

7. Rosenstein ED, Greenwald RA, Kushner LJ, Weissmann G. Hypothesis: the humoral immune response to oral bacteria provides a stimulus for the development of rheumatoid arthritis. Inflammation 2004;28:311-318.

8. de Pablo P, Dietrich T, McAlindon TE. Association of periodontal disease and tooth loss with rheumatoid arthritis in the US population. J Rheumatol 2008;35:70-76.

9. Georgiou TO, Marshall RI, Bartold PM. Prevalence of systemic diseases in Brisbane general and periodontal practice patients. Aust Dent J 2004;49:177-184.

10. Arnett FC, Edworthy SM, Bloch DA, et al. The American Rheumatism Association 1987 revised criteria for the classification of rheumatoid arthritis. Arthritis Rheum 1988;31:315-324.

11. Wegner N, Wait R, Sroka A, et al. Peptidylarginine deiminase from Porphyromonas gingivalis citrullinates human fibrinogen and $\alpha$-enolase: implications for autoimmunity in rheumatoid arthritis. Arthritis Rheum 2010;62:26622672.

12. Arvikar SL, Collier DS, Fisher MC, et al. Clinical correlations with Porphyromonas gingivalis antibody responses in patients with early rheumatoid arthritis. Arthritis Res Ther 2013;15:R109.

13. Lundberg K, Wegner N, Yucel-Lindberg T, Venables PJ. Periodontitis in RA-the citrullinated enolase connection. Nat Rev Rheumatol 2010;6:727-730.

14. Dissick A, Redman RS, Jones M, et al. Association of periodontitis with rheumatoid arthritis: a pilot study. J Periodontol 2010;81:223-230.

15. de Smit M, Westra J, Vissink A, et al. Periodontitis in established rheumatoid arthritis patients: a cross-sectional clinical, microbiological and serological study. Arthritis Res Ther 2012;14:R222.

16. Mikuls TR, Payne JB, Yu F, et al. Periodontitis and Porphyromonas gingivalis in patients with rheumatoid arthritis. Arthritis Rheumatol 2014;66:1090-1100.

17. Khantisopon N, Louthrenoo W, Kasitanon N, et al. Periodontal disease in Thai patients with rheumatoid arthritis. Int J Rheum Dis 2014;17:511-518.

18. Joseph R, Rajappan S, Nath SG, Paul BJ. Association between chronic periodontitis and rheumatoid arthritis: a hospital-based case-control study. Rheumatol Int 2013;33:103-109.

19. Vander Cruyssen B, Van Looy S, Wyns B, et al. DAS28 best reflects the physician's clinical judgment of response to 
infliximab therapy in rheumatoid arthritis patients: validation of the DAS28 score in patients under infliximab treatment. Arthritis Res Ther 2005;7:R1063-R1071.

20. Lundberg K, Kinloch A, Fisher BA, et al. Antibodies to citrullinated alpha-enolase peptide 1 are specific for rheumatoid arthritis and cross-react with bacterial enolase. Arthritis Rheum 2008;58:3009-3019.

21. Bannai M, Tokunaga $\mathrm{K}$, Lin L, et al. Discrimination of human HLA-DRB1 alleles by PCR-SSCP (single-strand conformation polymorphism) method. Eur J Immunogenet 1994;21:1-9.

22. Im CH, Kang EH, Ryu HJ, et al. Anti-cyclic citrullinated peptide antibody is associated with radiographic erosion in rheumatoid arthritis independently of shared epitope status. Rheumatol Int 2009;29:251-256.

23. Silness J, Loe H. Periodontal disease in pregnancy. II. Correlation between oral hygiene and periodontal condtion. Acta Odontol Scand 1964;22:121-135.

24. Loe H, Silness J. Periodontal disease in pregnancy. I. Prevalence and severity. Acta Odontol Scand 1963;21:533551.

25. Armitage GC. Development of a classification system for periodontal diseases and conditions. Ann Periodontol
1999;4:1-6.

26. Cantley MD, Haynes DR, Marino V, Bartold PM. Pre-existing periodontitis exacerbates experimental arthritis in a mouse model. J Clin Periodontol 2011;38:532-541.

27. Marchesan JT, Gerow EA, Schaff R, et al. Porphyromonas gingivalis oral infection exacerbates the development and severity of collagen-induced arthritis. Arthritis Res Ther 2013;15:R186.

28. de Pablo P, Dietrich T, Chapple IL, et al. The autoantibody repertoire in periodontitis: a role in the induction of autoimmunity to citrullinated proteins in rheumatoid arthritis? Ann Rheum Dis 2014;73:580-586.

29. American Dental Association Divison of Communications; Journal of the American Dental Association. For the dental patient: treating periodontal diseases. J Am Dent Assoc 2005;136:127.

30. Stastny P. Association of the B-cell alloantigen DRw4 with rheumatoid arthritis. N Engl J Med 1978;298:869-871.

31. Dougados M, Soubrier M, Antunez A, et al. Prevalence of comorbidities in rheumatoid arthritis and evaluation of their monitoring: results of an international, cross-sectional study (COMORA). Ann Rheum Dis 2014;73:62-68. 